\title{
Three-dimensional point spread functions of an optical heterodyne scanning image processor
}

\author{
Ting-Chung Poon and Guy Indebetouw
}

\begin{abstract}
We study the three-dimensional (3-D) imaging properties of an optical heterodyne scanning image processor. The image processor is a two-pupil optical system capable of 3-D imaging coherently or incoherently, depending on the detection scheme used. We derive the imaging properties in terms of the two pupils and then show an important 3-D imaging application in scanning holography by deriving its 3-D point spread functions and compare them with conventional 3-D imaging systems. (C) 2003 Optical Society of America
\end{abstract}

OCIS codes: $110.0110,090.0090$.

\section{Introduction}

Conventional incoherent image processing can only implement real and positive point spread function(s) (PSF)(s) which precludes some of the most useful linear operations, such as edge detection and deblurring. ${ }^{1}$ It is now known that two-pupil processing techniques can achieve bipolar or even complex $\mathrm{PSFs}^{2-7}$ in incoherent image processing. One of the two-pupil processing techniques, namely the use of a pupil interaction scheme in a scanning illumination mode, has been developed quite well.4,5,7 This technique has been implemented by an optical heterodyne scanning image processor and has been used for many interesting applications, such as textural edge extraction, and tunable and three-dimensional (3-D) filtering. ${ }^{8,9}$ Moreover, optical scanning holography has been invented to acquire holographic information through two-dimensional (2-D) optical scanning. ${ }^{10}$ Scanning holographic microscopy and 3-D optical remote sensing are some of its most recent applications. ${ }^{11,12}$ Some properties of a scanning holographic microscope have been outlined recently. ${ }^{13}$ Numerical simulations have shown that PSFs leading to different imaging functionalities (e.g., enhanced spatial resolution, extended depth of focus, or

T.-C. Poon is with the Bradley Department of Electrical and Computer Engineering and G. Indebetouw is with the Department of Physics, and both are with Virginia Polytechnic Institute and State University, Blacksburg, Virginia 24061.

Received 23 July 2002; revised manuscript received 10 October 2002.

0003-6935/03/081485-08\$15.00/0

(C) 2003 Optical Society of America optical sectioning) can be expected with proper choices of the pupil functions. ${ }^{14}$

The purpose of this paper is to investigate the $3-\mathrm{D}$ imaging properties in terms of the two pupils presented in the scanning image processor. The paper presents what is to our knowledge a new analysis of the PSF of the scanning processor based on Fourier optics. It focuses on the availability of different imaging modes (coherent and incoherent) that were not sufficiently stressed in previous papers. ${ }^{13,14}$ Indeed, as a result of the analysis, we show that, if one of the pupils is a clear mask, the 3-D PSFs in the incoherent as well as in the coherent case have the same form as the 3-D coherent PSF in conventional 3-D imaging, i.e., the 3-D PSF is given by the in-focus coherent PSF convolved by the free-space spatial impulse response in Fourier optics. We then use optical scanning holography as an example to derive its 3-D PSFs and compare them with those obtained with conventional 3-D holography. We also show that, depending on how we obtain the complex holograms, we can obtain reconstructions corresponding to the real and pseudoscopic reconstruction of a conventional hologram. In Section 2, we briefly discuss the 3-D imaging of a conventional 4- $f$ optical system and obtain its $3-\mathrm{D}$ PSFs under coherent and incoherent imaging. In Section 3, we derive 3-D imaging properties as functions of the two-pupil distributions associated with the system and discuss some examples pertaining to optical scanning holography. Finally, in Section 4, we make some concluding remarks.

\section{Conventional 3-D Imaging}

Figure 1 shows a conventional 4-f optical system. The system shows the imaging of a $3-\mathrm{D}$ object. The 


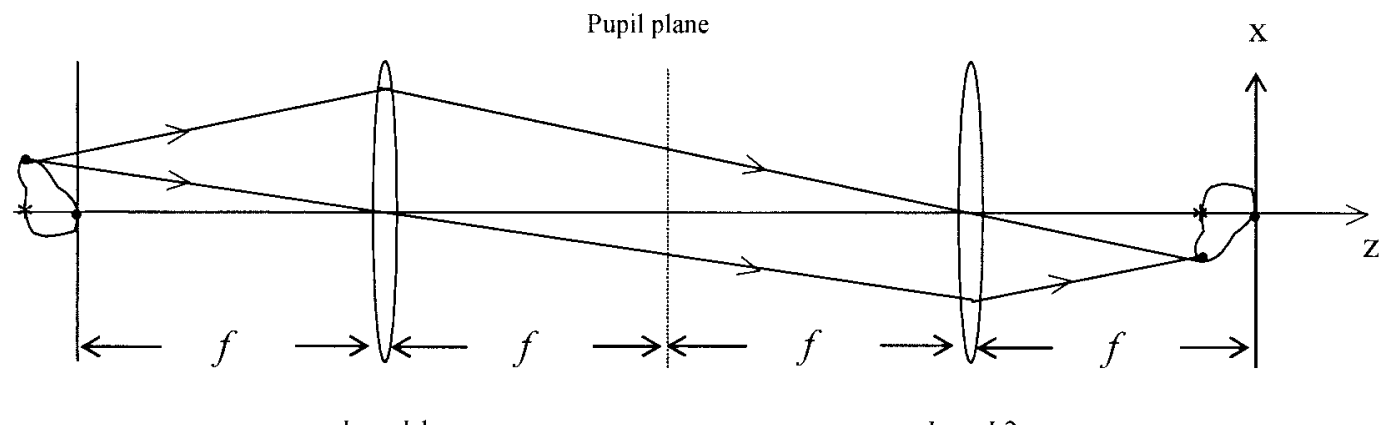

Lens L1

Lens L2

Fig. 1. Conventional 4- $f$ 3-D imaging system.

object is assumed to be a dilute (weakly scattering) transparent object, or a diffusely reflecting surface without shadowing or vignetting. Hence the derivations that follow are consistent with the well-known first-order Born's approximation. ${ }^{15}$ In this situation, the imaging is linear (or bilinear if partially coherent), and a PSF can be defined. ${ }^{16}$ To find the impulse response, we place a point source on the input plane, which is on the front focal plane of lens L1. At the output plane, which is the back focal plane of lens L2, the impulse response is given by $p(x$, $y$ ), which is the Fourier transform of the pupil function located on the pupil plane, in the coincident back focal plane of L1, and front focal plane of L2. If we let this response to propagate a distance $z$, we have a 3 -D coherent PSF, $\operatorname{CPSF}(x, y, z)=p(x, y) * h(x, y ; z)$, where $*$ denotes convolution and $h(x, y ; z)$ is the free-space spatial response in Fourier optics ${ }^{17}$ (also, see Appendix A. for definitions). If the illumination light source is incoherent, we take the squared magnitude of CPSF to obtain a 3-D incoherent or intensity point-spread function (IPSF), i.e., IPSF = $|\mathrm{CPSF}|^{2}$ (see Refs. 17 and 18).

\section{Two-Pupil Optical Heterodyne Scanning Processor}

\section{A. Basic Theory}

A typical two-pupil heterodyne optical scanning system is shown in Fig. 2. Along the horizontal direction, we see that the 3-D object now is placed between the two lenses L1 and L2 in contrast to 3-D imaging in Fig. 1, where the object is placed in the front focal plane of L1. The 3-D object is 2-D scanned by two co-propagating beams that have been modified by the two pupils located in the pupil planes as shown. The two optical beams have different temporal frequencies $\omega_{0}$ and $\omega_{0}+\Omega$ as indicated in the figure. $p_{1}(x$, $y$ ) is the impulse response, located on the back focal plane of lens L1, due to the beam of frequency $\omega_{0}$, and $p_{2}(x, y)$ is the impulse response due to the beam of frequency $\omega_{0}+\Omega$. The two impulse responses are combined by beam splitter BS and propagate over a distance $z_{0}+z$ to scan over a thin and weakly scattering object slide that is represented by an amplitude transmittance $T(x, y ; z)$, as shown in the figure. We model the 3-D object as a stack of transverse slices spanning a longitudinal range $\Delta z \ll z_{0}$, where
$\Delta z$ is the thickness of the 3-D object. Twodimensional scanning can be done by moving the 3-D object along the $x$ and $y$ directions while keeping the two beams fixed, or by moving the beams and the detector in synchronism. Mathematically, the amplitude distributions of the two beams just before the slice at position $z$ is given by $P_{1}\left(x, y ; z+z_{0}\right) \exp \left(j \omega_{0} t\right)$ $+P_{2}\left(x, y ; z+z_{0}\right) \exp \left[j\left(\omega_{0}+\Omega t\right)\right]$, where $P_{i}(x, y ; z+$ $\left.z_{0}\right)=p_{i}(x, y) * h\left(x, y ; z+z_{0}\right)$ with $i=1$ or 2 . The field just after the object slice is $\left\{P_{1}\left(x^{\prime}, y^{\prime} ; z+\right.\right.$ $\left.\left.z_{0}\right) \exp \left(j \omega_{0} t\right)+P_{2}\left(x^{\prime}, y^{\prime} ; z+z_{0}\right) \exp \left[j\left(\omega_{0}+\Omega t\right)\right]\right\} T\left(x^{\prime}+\right.$ $\left.x, y^{\prime}+y ; z\right)$, where $x=x(t)$ and $y=y(t)$ represent the instantaneous 2-D position of the object. This field then propagates through the Fourier transform lens L2 and reaches the mask, $M(x, y)$, located in the back focal plane of lens L2. Hence the field distribution exiting from the mask, due to all the slices of the object $T(x, y ; z)$, is

$$
\begin{aligned}
\psi\left(x, y ; x_{m}, y_{m}\right) \propto & \left(\int \left\{P_{1}\left(x^{\prime}, y^{\prime} ; z+z_{0}\right) \exp \left(j \omega_{0} t\right)\right.\right. \\
& +P_{2}\left(x^{\prime}, y^{\prime} ; z+z_{0}\right) \exp [j \\
& \left.\left.\times\left(\omega_{0}+\Omega t\right)\right]\right\} T\left(x^{\prime}+x, y^{\prime}+y ; z\right) \\
& \times \exp \left[j \frac{k_{0}}{f}\left(x^{\prime} x_{m}+y^{\prime} y_{m}\right)\right] \\
& \times \mathrm{d} x^{\prime} \mathrm{d} y^{\prime} \exp \left[-j \frac{k_{0} z}{2 f^{2}}\right. \\
& \left.\left.\times\left(x_{m}{ }^{2}+y_{m}{ }^{2}\right)\right] \mathrm{d} z\right) M\left(x_{m}, y_{m}\right),
\end{aligned}
$$

where $x_{m}$ and $y_{m}$ are the coordinates in the plane of the mask, $k_{0}=2 \pi / \lambda$ is the wavenumber, and $z$ is the distance of an object slice measured from the front focal plane of lens L2. The integration over $z$ represents the volumetric effect due to the 3 -D object. Finally, the photodetector, PD, which responds to intensity, gives the current output $i(x, y)$ by spatially integrating the intensity:

$$
i(x, y) \propto \int\left|\psi\left(x, y ; x_{m}, y_{m}\right)\right|^{2} \mathrm{~d} x_{m} \mathrm{~d} y_{m},
$$




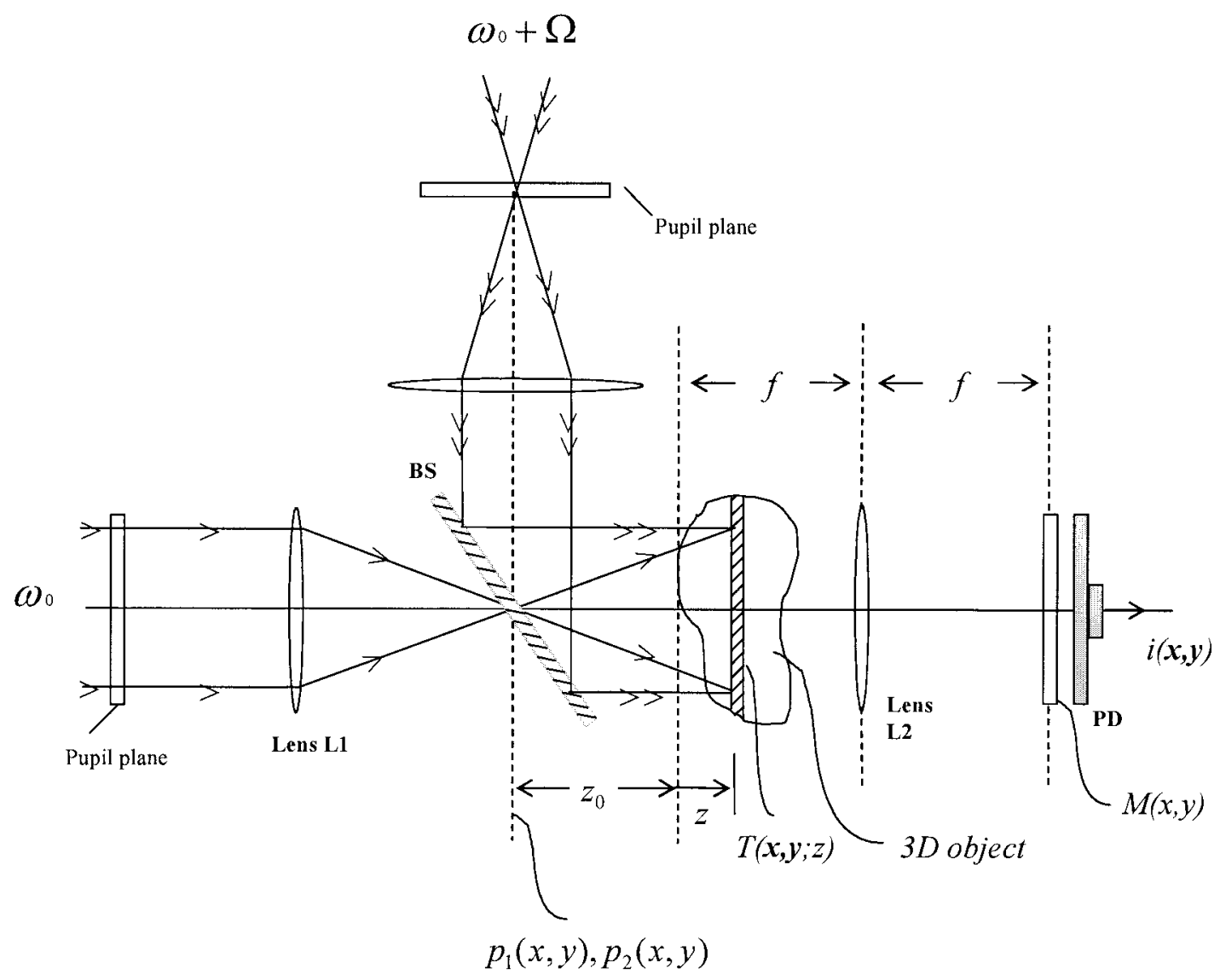

Fig. 2. Two-pupil heterodyne scanning processor.

where the integration is over the area of the photodetector. $i(x, y)$ consists of a baseband current and a heterodyne current at frequency $\Omega$. After some manipulations, the heterodyne current $i_{\Omega}(x, y)$ is given by

$$
\begin{aligned}
i_{\Omega}(x, y) \propto & \int\left[P_{1}\left(x^{\prime}, y^{\prime} ; z^{\prime}+z_{0}\right) P_{2}^{*}\left(x^{\prime \prime}, y^{\prime \prime} ; z^{\prime \prime}+z_{0}\right)\right. \\
& \times \exp (-j \Omega t)+P_{2}\left(x^{\prime}, y^{\prime} ; z^{\prime}+z_{0}\right) \\
& \times P_{1}^{*}\left(x^{\prime \prime}, y^{\prime \prime} ; z^{\prime \prime}+z_{0}\right) \\
& \times \exp (j \Omega t)] \exp \left\{j \frac { k _ { 0 } } { f } \left[x_{m}\left(x^{\prime}-x^{\prime \prime}\right)\right.\right. \\
& \left.\left.+y_{m}\left(y^{\prime}-y^{\prime \prime}\right)\right]\right\} \exp \left[-j \frac{k_{0}\left(z^{\prime}-z^{\prime \prime}\right)}{2 f^{2}}\right. \\
& \left.\times\left(x_{m}{ }^{2}+y_{m}{ }^{2}\right)\right] T\left(x^{\prime}+x, y^{\prime}+y ; z^{\prime}\right) \\
& \times T^{*}\left(x^{\prime \prime}+x, y^{\prime \prime}+y ; z^{\prime \prime}\right) \\
& \times\left|M\left(x_{m}, y_{m}\right)\right|^{2} \\
& \times \mathrm{d} x^{\prime} \mathrm{d} y^{\prime} \mathrm{d} x^{\prime \prime} \mathrm{d} y^{\prime \prime} \mathrm{d} z^{\prime} \mathrm{d} z^{\prime \prime} \mathrm{d} x_{m} \mathrm{~d} y_{m} .
\end{aligned}
$$

For the special but important cases of selfluminous objects, such as fluorescent specimens or incoherently reflecting rough surfaces as encountered in remote sensing, the lens L2 serves the simple pur- pose of collecting most of the energy scattered by the object. ${ }^{12,19}$ This energy integrated by the detector is thus equivalent to the integrated energy immediately behind the object. Under this situation, the scanned current becomes

$$
\begin{aligned}
i(x, y) \propto & \int\left\{P_{1}\left(x^{\prime}, y^{\prime} ; z+z_{0}\right) \exp \left(j \omega_{0} t\right)\right. \\
& +P_{2}\left(x^{\prime}, y^{\prime} ; z+z_{0}\right) \exp \left[j\left(\omega_{0}+\Omega t\right]\right\} \\
& \times\left|T\left(x^{\prime}+x, y^{\prime}+y ; z\right)\right|^{2} \mathrm{~d} z \mathrm{~d} x^{\prime} \mathrm{d} y^{\prime} .
\end{aligned}
$$

The integration is over the photodetector's active surface, which is assumed sufficiently large. The heterodyne current is given by

$$
\begin{aligned}
i_{\Omega}(x, y) \propto & \operatorname{Re}\left[\int P_{1} *\left(x^{\prime}, y^{\prime} ; z+z_{0}\right) P_{2}\left(x^{\prime}, y^{\prime} ; z+z_{0}\right)\right. \\
& \times\left|T\left(x^{\prime}+x, y^{\prime}+y ; z\right)\right|^{2} \mathrm{~d} x^{\prime} \mathrm{d} y^{\prime} \mathrm{d} z \\
& \times \exp (j \Omega t)]
\end{aligned}
$$

where $\mathrm{Re}[$.$] represents the real part of the quantity$ being bracketed. Note that, from (5), only the intensity distribution is processed. Equations (3) and (5) are the core results of the heterodyne scanning processor and they represent scanned and processed [by $p_{1}(x$, $y$ ) and $\left.p_{2}(x, y)\right]$ versions of the 3 -D object $T(x, y ; z)$ or 


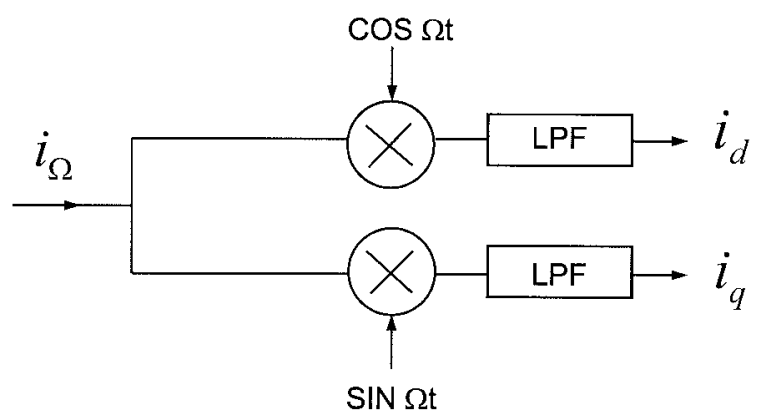

Fig. 3. Electronic demodulation system.

$|T(x, y ; z)|^{2}$, depending on the situation encountered. In the next section, we discuss some special cases.

B. Point Spread Function of three-dimensional imaging

Equation (5) has been applied for most of the applications encountered thus far with the heterodyne scanning processor, e.g., fluorescence microscopy and remote sensing. ${ }^{11,12,19}$ In this section, we discuss 3-D imaging based on Eq. (5) in the case of optical scanning holography, ${ }^{10}$ and compare it with conventional 3-D imaging by deriving its 3-D PSFs.

The processed information can be obtained from Eq. (5) by using a number of demodulation or filtering schemes. For example, we can rewrite Eq. (5) as

$$
\begin{aligned}
i_{\Omega}(x, y) & \propto \operatorname{Re}\left[i_{\Omega_{p}}(x, y) \exp (j \Omega t)\right] \\
& =\left|i_{\Omega_{p}}(x, y)\right| \cos (\Omega t+\theta),
\end{aligned}
$$

where $i_{\Omega_{p}}(x, y)=\int P_{1}^{*}\left(x^{\prime}, y^{\prime} ; z+z_{0}\right) P_{2}\left(x^{\prime}, y^{\prime} ; z+\right.$ $\left.z_{0}\right)\left|T\left(x^{\prime}+x, y^{\prime}+y ; z\right)\right|^{2} \mathrm{~d} x^{\prime} \mathrm{d} y^{\prime} \mathrm{d} z$. When $i_{\Omega}(x, y)$ is demodulated according to the scheme shown in Fig. 3, where LPF denotes electronic lowpass filtering, we have two demodulated currents: $i_{d}(x, y)=\mid i_{\Omega_{p}}(x$, $y) \mid \cos (\theta)$ and $i_{q}(x, y)=-\left|i_{\Omega_{p}}(x, y)\right| \sin (\theta)$. When these currents are displayed in synchronization with the $\mathrm{x}-\mathrm{y}$-scanner, we have two processed images of $|T|^{2}$. If we further perform the complex addition of the two currents $i_{\mathrm{d}}(x, y)$ and $i_{q}(x, y)$, we can recover the processed complex information

$$
i_{\mathrm{d}}(x, y)-j i_{q}(x, y)=i_{\Omega_{p}}(x, y) .
$$

The complex addition is conveniently done by a digital computer. Interestingly, the complex conjugate of $i_{\Omega_{p}}(x, y)$, i.e., $i_{\Omega_{p}}^{*}(x, y)$ can be obtained if we use $+j$ instead of $-j$ in the above addition. This leads to a different physical interpretation of the processed images as is shown in the following simple example.

We have a choice of $p_{1}(x, y)$ and $p_{2}(x, y)$ to process our information, and for simplicity, let us choose $p_{2}(x$, $y)=1$ and leave $p_{1}(x, y)$ as an arbitrary distribution. From Eq. (6) $i_{\Omega_{p}}(x, y)$ then becomes

$$
\begin{aligned}
i_{\Omega_{p}}(x, y)= & \int\left[p_{1}\left(x^{\prime}, y^{\prime}\right) * h\left(x^{\prime}, y^{\prime} ; z+z_{0}\right)\right]^{*} \\
& \times\left.\exp \left[-j k_{0}\left(z+z_{0}\right)\right] T\left(x^{\prime}+x, y^{\prime}+y ; z\right)\right|^{2} \\
& \times \mathrm{d} x^{\prime} \mathrm{d} y^{\prime} \mathrm{d} z
\end{aligned}
$$

Again, $i_{\Omega_{p}}(x, y)$ is a 2-D record of the 3-D information. If we display this using a spatial light modulator (SLM), upon illumination by a plane wave we have the field distribution at a distance $z$ away, given by (after some manipulations)

$$
\begin{aligned}
i_{\Omega_{p}}(x, y) * h(x, y ; z)= & \exp \left(-j k_{0} z_{0}\right) \\
& \times\left\{\left[p_{1} *(-x,-y) * h(x, y ; z\right.\right. \\
& \left.\left.-z_{0}\right)\right] * *\left[|T(x, y ; z)|^{2}\right. \\
& \left.\left.\times \exp \left(-j k_{0} z\right)\right]\right\}
\end{aligned}
$$

where $* *$ denotes a 3-D convolution involving $x, y$, and $z$ (see Appendix A. for definition). If we use $i_{\Omega_{p}} *(x, y)$ instead to be displayed on the SLM, we obtain

$$
\begin{aligned}
i_{\Omega_{p}} *(x, y) * h^{*}(x, y ; z)= & \exp \left(j k_{0} z_{0}\right) \\
& \times\left\{\left[p_{1}(-x,-y) * h^{*}(x, y ; z\right.\right. \\
& \left.\left.-z_{0}\right)\right] * *\left[|T(x, y ; z)|^{2}\right. \\
& \left.\left.\times \exp \left(j k_{0} z\right)\right]\right\} .
\end{aligned}
$$

The 3-D PSF of the above systems can be found by taking $|T(x, y ; z)|^{2}=\delta(x, y ; z)$. Eqs. (9) and (10) become the 3-D incoherent PSFs

$$
\begin{aligned}
& \operatorname{IPSF}(x, y, z)=\left[p_{1}^{*}(-x,-y) * h\left(x, y ; z-z_{0}\right)\right], \\
& \operatorname{IPSF}(x, y, z)=\left[p_{1}(-x,-y) * h^{*}\left(x, y ; z-z_{0}\right)\right],
\end{aligned}
$$

respectively. These PSFs have the same form as the 3-D coherent PSF in conventional 3-D imaging as discussed in Section 2. To clarify these results, let us take optical scanning holography as an example. In optical scanning holography, the object is scanned by $p_{1}(x, y)=\delta(x, y)$ and $p_{2}(x, y)=1$, and hence the 3-D PSFs are $h\left(x, y ; z-z_{0}\right)$ and $h^{*}\left(x, y ; z-z_{0}\right)$, respectively, for the two cases. Let us first look at the first case. We assume that we record three point sources as shown in Fig. 4. The 3-D object is then given by $|T(x, y ; z)|^{2}=\delta(x, y, z)+\delta\left(x, y, z-z_{1}\right)+\delta\left(x+x_{1}, y\right.$, $\left.z-z_{1}\right)$. What is being recorded as a hologram is then given by Eq. (8). Upon plane wave illumination of the data displayed on an SLM, we obtain, from Eq. (9),

$$
\begin{aligned}
i_{\Omega_{p}}(x, y) * h(x, y ; z)= & h\left(x, y ; z-z_{0}\right) \\
& +\exp \left(-j k_{0} z_{1}\right) h\left(x, y ; z-z_{1}\right. \\
& \left.-z_{0}\right)+\exp \left(-j k_{0} z_{1}\right) h(x \\
& \left.+x_{1}, y ; z-z_{1}-z_{0}\right) .
\end{aligned}
$$

Physically, this corresponds to a reconstruction of three point sources as shown in Fig. 5(a). For the case of Eq. (10), we obtain

$$
\begin{aligned}
i_{\Omega_{p}}^{*}(x, y) * h^{*}(x, y ; z)= & h^{*}\left(x, y ; z-z_{0}\right) \\
& +\exp \left(j k_{0} z_{1}\right) h^{*}\left(x, y ; z-z_{1}\right. \\
& \left.-z_{0}\right)+\exp \left(j k_{0} z_{1}\right) h^{*}(x \\
& \left.+x_{1}, y ; z-z_{1}-z_{0}\right),
\end{aligned}
$$




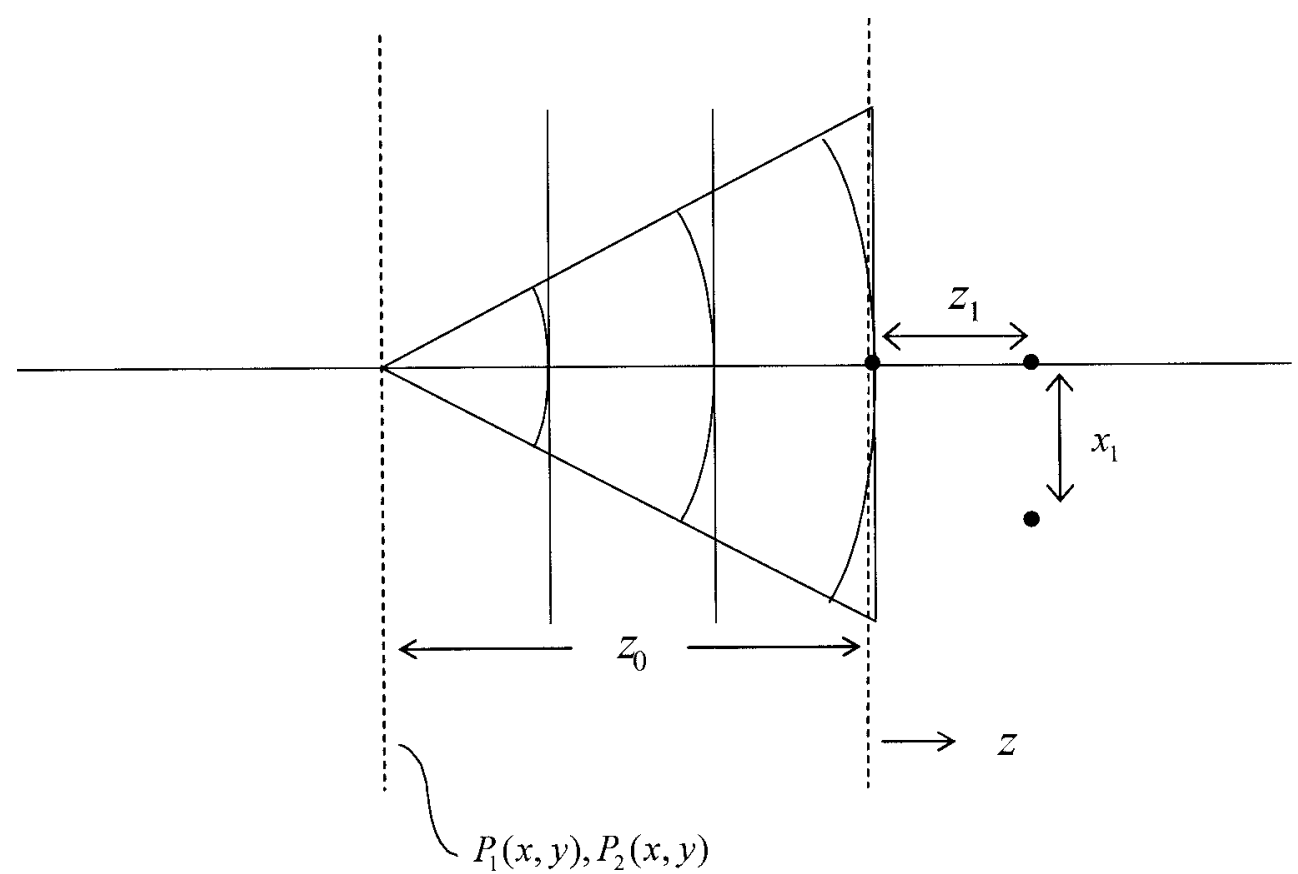

Fig. 4. Optical scanning holographic recording of a 3-point source.

and this corresponds to the reconstruction of 3 virtual point sources as shown in Fig. 5(b). These two conjugate reconstructions correspond to the real and pseudoscopic reconstructions of a conventional hologram, respectively.

\section{Coherency of the Processor}

In this section, we investigate the coherency of the processor by investigating a special case of Eq. (3) in greater details. For a pinhole mask centered on the axis, we let $M(x, y)=\delta(x, y)$. After integrating over the mask's coordinates, Eq. (3) becomes

$$
\begin{aligned}
i_{\Omega}^{\delta}(x, y) \propto & \int\left[P_{1}\left(x^{\prime}, y^{\prime} ; z^{\prime}+z_{0}\right) P_{2}^{*}\left(x^{\prime \prime}, y^{\prime \prime} ; z^{\prime \prime}+z_{0}\right)\right. \\
& \times \exp (-j \Omega t)+P_{2}\left(x^{\prime}, y^{\prime} ; z^{\prime}+z_{0}\right) \\
& \left.\times P_{1}^{*}\left(x^{\prime \prime}, y^{\prime \prime} ; z^{\prime \prime}+z_{0}\right) \exp (j \Omega t)\right] \\
& \times T\left(x^{\prime}+x, y^{\prime}+y ; z^{\prime}\right) T^{*}\left(x^{\prime \prime}+x, y^{\prime \prime}\right. \\
& \left.+y ; z^{\prime \prime}\right) \mathrm{d} x^{\prime} \mathrm{d} y^{\prime} \mathrm{d} x^{\prime \prime} \mathrm{d} y^{\prime \prime} \mathrm{d} z^{\prime} \mathrm{d} z^{\prime \prime}
\end{aligned}
$$

Rearranging relation (14), we have

$$
\begin{aligned}
i_{\Omega}^{\delta}(x, y) \propto & \operatorname{Re}\left\{\int \left[P _ { 2 } ( x ^ { \prime } , y ^ { \prime } ; z ^ { \prime } + z _ { 0 } ) T \left(x^{\prime}+x, y^{\prime}\right.\right.\right. \\
& \left.\left.+y ; z^{\prime}\right) \mathrm{d} x^{\prime} \mathrm{d} y^{\prime} \mathrm{d} z^{\prime}\right] \int\left[P _ { 1 } ^ { * } \left(x^{\prime \prime}, y^{\prime \prime} ; z^{\prime \prime}\right.\right. \\
& \left.+z_{0}\right) T^{*}\left(x^{\prime \prime}+x, y^{\prime \prime}+y ; z^{\prime \prime}\right) \mathrm{d} x^{\prime \prime} \mathrm{d} y^{\prime \prime} \mathrm{d} z^{\prime \prime} \\
& \times \exp (j \Omega t)]\}
\end{aligned}
$$

Again for simplicity, we let $p_{1}(x, y)=1$ and leave $p_{2}(x, y)$ as is. Under this situation, $\int P_{1}^{*}\left(x^{\prime \prime}, y^{\prime \prime} ; z^{\prime \prime}+\right.$ $\left.z_{0}\right) T^{*}\left(x^{\prime \prime}+x, y^{\prime \prime}+y ; z^{\prime \prime}\right) \mathrm{d} x^{\prime \prime} \mathrm{d} y^{\prime \prime} \mathrm{d} z^{\prime \prime}$ is a constant, and relation (15) becomes

$$
i_{\Omega}^{\delta}(x, y) \propto \operatorname{Re}\left[i_{\Omega_{p}}{ }^{\delta}(x, y) \exp (j \Omega t)\right],
$$

where $i_{\Omega_{p}}{ }^{\delta}(x, y)=\int\left[P_{2}\left(x^{\prime}, y^{\prime} ; z^{\prime}+z_{0}\right) T\left(x^{\prime}+x, y^{\prime}+\right.\right.$ $\left.\left.y ; z^{\prime}\right) \mathrm{d} x^{\prime} \mathrm{d} y^{\prime} \mathrm{d} z^{\prime}\right]$. Eq. (16) has the same form as Eq. (6) but we notice that we can now process the object's amplitude transmittance rather than the intensity. Moreover, the processing is only done by one pupil, namely $p_{2}(x, y)$. Following the result of the last section, we see that if again we use Fig. 3 for demodulation to construct a complex transmittance for SLM reconstruction, the 3-D coherent PSF for the system becomes

$$
\operatorname{CPSF}(x, y, z)=\left[p_{2}(-x,-y) * h *\left(x, y ; z-z_{0}\right)\right] .
$$

D. Incoherent Processing

Eq. (3) can be written as

$$
\begin{aligned}
& \int\left[P_{1}\left(x^{\prime}, y^{\prime} ; z^{\prime}+z_{0}\right) P_{2}^{*}\left(x^{\prime \prime}, y^{\prime \prime} ; z^{\prime \prime}+z_{0}\right) \exp (-j \Omega t)\right. \\
& \left.+P_{2}\left(x^{\prime}, y^{\prime} ; z^{\prime}+z_{0}\right) P_{1}^{*}\left(x^{\prime \prime}, y^{\prime \prime} ; z^{\prime \prime}+z_{0}\right) \exp (j \Omega t)\right] \\
& \times T\left(x^{\prime}+x, y^{\prime}+y ; z^{\prime}\right) T^{*}\left(x^{\prime \prime}+x, y^{\prime \prime}+y ; z^{\prime \prime}\right) \Gamma\left(x^{\prime}\right. \\
& \left.\quad-x^{\prime \prime}, y^{\prime}-y^{\prime \prime}, z^{\prime}-z^{\prime \prime}\right) \mathrm{d} x^{\prime} \mathrm{d} y^{\prime} \mathrm{d} x^{\prime \prime} \mathrm{d} y^{\prime \prime} \mathrm{d} z^{\prime} \mathrm{d} z^{\prime \prime},
\end{aligned}
$$




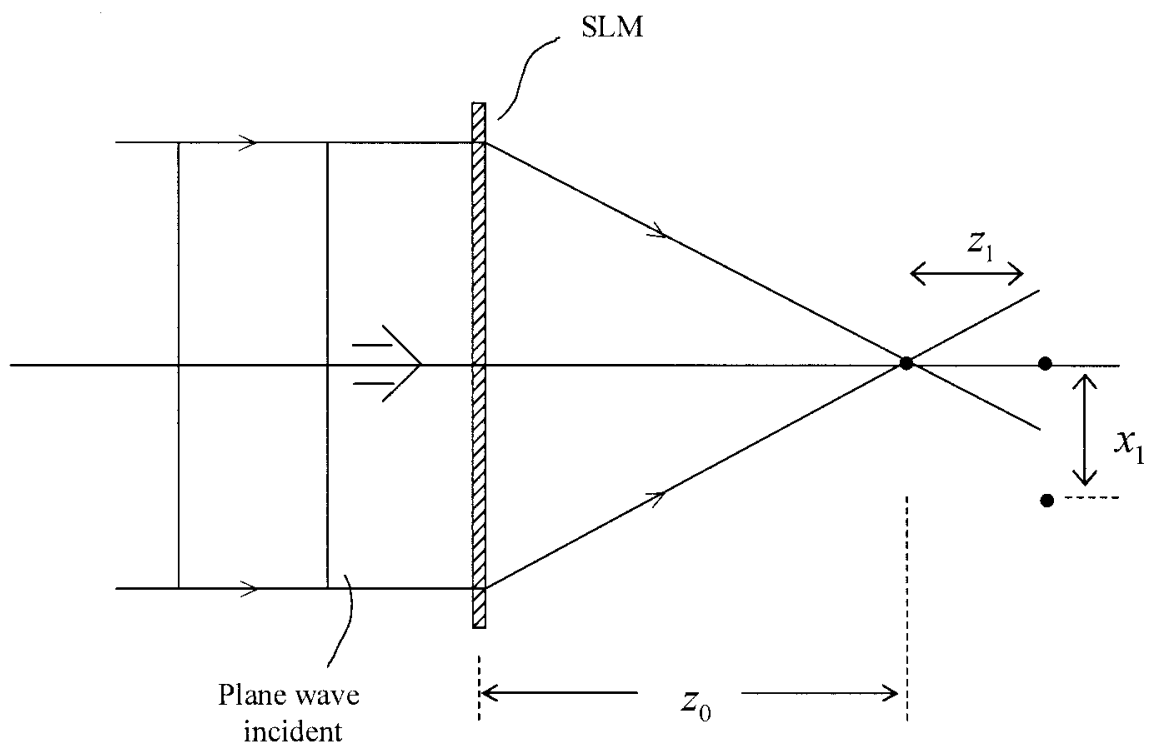

(a)

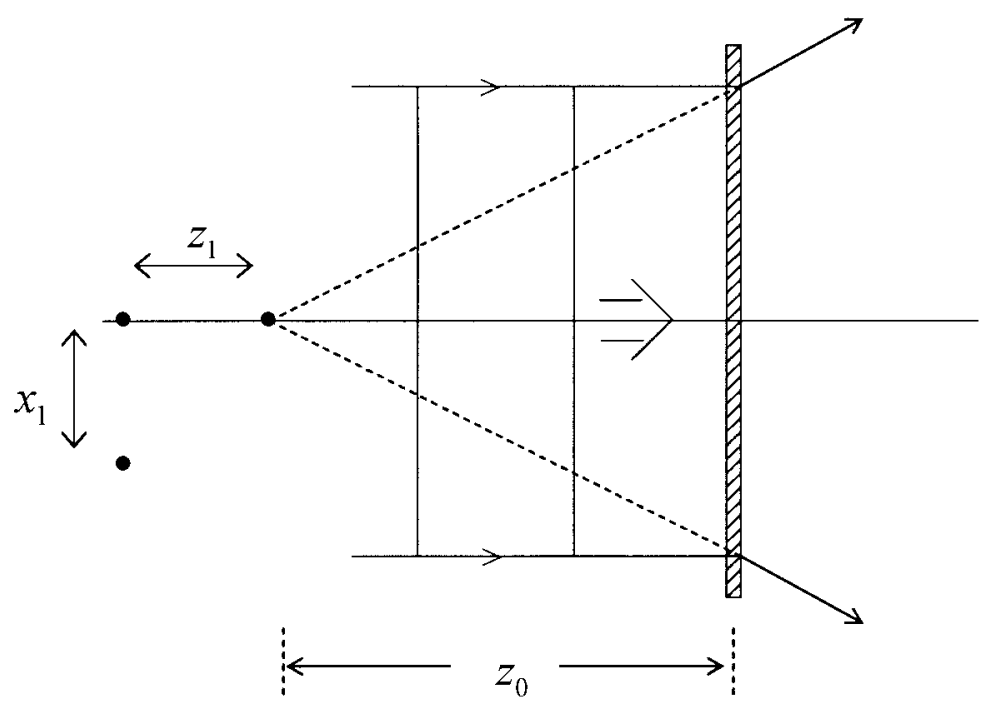

(b)

Fig. 5. (a) Holographic reconstruction when $i_{d}(x, y)-j i_{Q}(x, y)$ is used for constructing a complex transmittance for SLM display, (b) holographic reconstruction when $i_{d}(x, y)+j i_{Q}(x, y)$ is used for constructing a complex transmittance for SLM display.

where

$$
\begin{aligned}
& \Gamma\left(x^{\prime}-x^{\prime \prime}, y^{\prime}-y^{\prime \prime}, z^{\prime}-z^{\prime \prime}\right)=\int\left|M\left(x_{m}, y_{m}\right)\right|^{2} \\
& \quad \times \exp \left\{j \frac{k_{0}}{f}\left[x_{m}\left(x^{\prime}-x^{\prime \prime}\right)+y_{m}\left(y^{\prime}-y^{\prime \prime}\right)\right]\right\} \\
& \quad \times \exp \left[-j \frac{k_{0}\left(z^{\prime}-z^{\prime \prime}\right)}{2 f^{2}}\left(x_{m}{ }^{2}+y_{m}{ }^{2}\right)\right] \mathrm{d} x_{m} \mathrm{~d} y_{m} .
\end{aligned}
$$

The function $\Gamma\left(x^{\prime}-x^{\prime \prime}, y^{\prime}-y^{\prime \prime}, z^{\prime}-z^{\prime \prime}\right)$ plays the same role in scanning imaging as the coherence function does in conventional partially coherent imaging. It can thus be interpreted as a measure of the correla- tion of the field at $\left(x^{\prime}, y^{\prime}, z^{\prime}\right)$, and the field at $\left(x^{\prime \prime}, y^{\prime \prime}\right.$, $z^{\prime \prime}$.

In the example of the previous paragraph, we had $M\left(x_{m}, y_{m}\right)=\delta\left(x_{m}, y_{m}\right)$, leading to $\Gamma\left(x^{\prime}-x^{\prime \prime}, y^{\prime}-y^{\prime \prime}\right.$, $\left.z^{\prime}-z^{\prime \prime}\right)=1$, which corresponds to a coherent processing system. If we now let the mask become an open mask, we have $M\left(x_{m}, y_{m}\right)=1$, and we obtain

$$
\begin{aligned}
& \Gamma\left(x^{\prime}-x^{\prime \prime}, y^{\prime}-y^{\prime \prime}, z^{\prime}-z^{\prime \prime}\right)= \\
& \quad \int \exp \left\{j \frac{k_{0}}{f}\left[x_{m}\left(x^{\prime}-x^{\prime \prime}\right)+y_{m}\left(y^{\prime}-y^{\prime \prime}\right)\right]\right\} \\
& \quad \times \exp \left[-j \frac{k_{0}\left(z^{\prime}-z^{\prime \prime}\right)}{2 f^{2}}\left(x_{m}{ }^{2}+y_{m}{ }^{2}\right)\right] \mathrm{d} x_{m} \mathrm{~d} y_{m} .
\end{aligned}
$$


The quadratic term in Eq. (20) represents a spherical wave with a radius of curvature $R=f^{2} /\left(z^{\prime}-z^{\prime \prime}\right)$, which can be made arbitrarily large. Thus

$$
\begin{aligned}
\Gamma\left(x^{\prime}-x^{\prime \prime}, y^{\prime}-y^{\prime \prime}, z^{\prime}-z^{\prime \prime}\right) \sim \frac{1}{z^{\prime}-z^{\prime \prime}} \\
\times \exp \left\{\frac{j k_{0}\left[\left(x^{\prime}-x^{\prime \prime}\right)^{2}+\left(y^{\prime}-y^{\prime \prime}\right)^{2}\right]}{2\left(z^{\prime}-z^{\prime \prime}\right)}\right\} \\
\sim \delta\left(x^{\prime}-x^{\prime \prime}, y^{\prime}-y^{\prime \prime} ; z^{\prime}-z^{\prime \prime}\right) .
\end{aligned}
$$

By substituting this result into Eq. (18), we obtain

$$
\begin{aligned}
i_{\Omega}(x, y) \propto & \operatorname{Re}\left[\int P_{1} *\left(x^{\prime}, y^{\prime} ; z+z_{0}\right) P_{2}\left(x^{\prime}, y^{\prime} ; z+z_{0}\right)\right. \\
& \times \mid T\left(x^{\prime}+x, y^{\prime}\right. \\
& \left.+y ; z)\left.\right|^{2} \mathrm{~d} x^{\prime} \mathrm{d} y^{\prime} \mathrm{d} z \exp (j \Omega t)\right]
\end{aligned}
$$

which is the same as Eq. (5), and corresponds to an incoherent processing system. By varying the size of the mask, it is thus possible to obtain images corresponding to various degrees of spatial coherence.

\section{Concluding Remarks}

We have developed a 3-D imaging theory for the twopupil optical heterodyne scanning image processor. The theory is applicable to dilute transparent $3-\mathrm{D}$ objects or to dilute diffusely reflecting surfaces without shadowing or vignetting. The processor can be used to process 3-D objects coherently or incoherently, depending on the detection scheme used. While the incoherent mode of operation has been applied for most of the applications, such as in 3-D fluorescence microscopy and in remote sensing, the coherent mode of operation so far has been limited to theory development. Coherent 3-D imaging, nevertheless, is an important extension of the processor in biological imaging for such an area as quantitative phase-contrast imaging. We have shown that the 3 -D imaging properties of the processor can be expressed in terms of the two pupils in the system. As a simple example, we have chosen one of the pupils as a uniform function. Subsequently, we have derived 3 -D coherent and incoherent point-spread functions (PSFs) and have shown that these PSFs have the same form as the 3-D coherent PSF in the conventional 4- $f$ imaging system. These important results show that bipolar or even complex PSFs are not only limited to 2-D bipolar incoherent processing but also in 3-D bipolar incoherent image processing. 3-D bipolar incoherent image processing remains a virtually unexplored topic. Last, we have illustrated one of its important 3-D imaging examples in optical scanning holography and shown that two reconstructions can be obtained corresponding to the real and pseudoscopic reconstructions of a conventional hologram.

\section{Appendix A.}

2-D convolution:

$$
\begin{aligned}
g_{1}(x, y) * g_{2}(x, y)= & \iint g_{1}\left(x^{\prime}, y^{\prime}\right) \\
& \times g_{2}\left(x-x^{\prime}, y-y^{\prime}\right) \mathrm{d} x^{\prime} \mathrm{d} y^{\prime} .
\end{aligned}
$$

3-D convolution:

$$
\begin{aligned}
g_{1}(x, y, z) * * g_{2}(x, y, z)= & \iiint g_{1}\left(x^{\prime}, y^{\prime}, z^{\prime}\right) \\
& \times g_{2}\left(x-x^{\prime}, y-y^{\prime},\right. \\
& \left.z-z^{\prime}\right) \mathrm{d} x^{\prime} \mathrm{d} y^{\prime} \mathrm{d} z^{\prime} .
\end{aligned}
$$

Free-space spatial impulse response in Fourier optics:

$$
h(x, y ; z)=\exp \left(-j k_{0} z\right) \frac{j k_{0}}{2 \pi z} \exp \left[-j \frac{k_{0}}{2 z}\left(x^{2}+y^{2}\right)\right] .
$$

T.-C. Poon acknowledges the support of the National Science Foundation (Grant \#ECS-9810158).

\section{References}

1. W. Lukosz, "Properties of linear low-pass filters for nonnegative signals," J. Opt. Soc. Am. 52, 827-829 (1962).

2. A. W. Lohmann and W. T. Rhodes, "Two-pupil synthesis of optical transfer functions," Appl. Opt. 17, 1141-1150 (1978).

3. W. Stoner, "Incoherent optical processing via spatially offset pupil masks," Opt. Appl. 17, 2454-2466 (1978).

4. T.-C. Poon and A. Korpel, "Optical transfer function of an acousto-optic heterodyning image processor," Opt. Lett. 4, 317-319 (1979).

5. T.-C. Poon, "Scanning holography and two-dimensional image processing by acousto-optic two-pupil synthesis," J. Opt. Soc. Am. A 2, 621-627 (1985).

6. J. N. Mait, "Pupil-function design for complex incoherent spatial filtering," J. Opt. Soc. Am. A 4, 1185-1193 (1987).

7. G. Indebetouw and T.-C. Poon, "Novel approaches of incoherent image processing with emphasis on scanning methods," Opt. Eng. 31, 2159-2167 (1992).

8. T.-C. Poon, J. Park, and G. Indebetouw, "Optical realization of textural edge extraction,” Opt. Commun. 65, 1-6 (1988).

9. T.-C. Poon, J. Park, and G. Indebetouw, "Real-time tunable incoherent spatial filtering: two-pupil processing technique," Opt. Eng. 29, 1507-1510 (1990).

10. T.-C. Poon, M. Wu, K. Shinoda, and Y. Suzuki, "Optical scanning holography," Proc. IEEE 84, 753-764 (1996).

11. T.-C. Poon, K. Doh, B. Schilling, M. Wu, K. Shinoda, and Y. Suzuki, "Three-dimensional microscopy by optical scanning holography," Opt. Eng. 34, 1338-1344 (1995).

12. P. Klysubun, G. Indebetpow, T. Kim, and T.-C. Poon, "Accuracy of 3-D remote target location using scanning holographic correlation," Opt. Commun. 184, 357-366 (2000).

13. G. Indebetouw, P. Klysubun, T. Kim, and T.-C. Poon, "Imaging properties of scanning holographic microscopy," J. Opt. Soc. Am. A 17, 380-390 (2000).

14. G. Indebetouw, "Properties of a scanning holographic microscope: improved resolution, extended depth-of-focus, and/or optical sectioning," J. Mod. Opt. 49, 1479-1500 (2002). 
15. M. Born and E. Wolf, Principles of Optics, 7th ed. p. 695-ff, (Cambridge University, Edinburgh, U.K., 1999), pp. 699-703.

16. B. J. Thompson, "Image formation with partially coherent light," in Progress in Optics, (E. Wolf, ed.), North-Holland, Amsterdam, 1969), Vol. 2.

17. T.-C. Poon and P. P. Banerjee, Contemporary Optical Image
Processing With Matlab (Elsevier Science, Oxford, U.K., 2001). 18. J. W. Goodman, Introduction to Fourier Optics, 2nd ed. (McGraw-Hill, New York, 1996).

19. B. Schilling, T.-C. Poon, G. Indebetouw, B. Storrie, K. Shinoda, and $\mathrm{M}$. Wu, "Three-dimensional holographic fluorescence microscopy," Opt. Lett. 22, 1506-1508 (1997). 